\title{
Sirenomelia
}

\section{Reporte de un caso}

Avila-Arambula $R^{* *}$, Obregón-Mimbela J***, Rosas-Cabral A.*

\section{Resumen}

La sirenomelia es una malformación congénita y suele ser letal debido a malformaciones asociadas, de etiología desconocida sin un patrón hereditario ni base genética demostrada. Se produce por una alteración temprana en el desarrollo vascular y el grado de defectos depende del momento en que se produce el secuestro sanguíneo durante la embriogénesis. Se puede asociar a malformaciones gastrointestinales, del aparato urinario y dextrocardia. En la antigüedad, los niños-sirena eran considerados "monstruos", y como tales eran asesinados o escondidos por su familia. Actualmente este tipo de malformación es fácilmente detectable a través de los exámenes de laboratorio y gabinete que en la actualidad se tiene. Se presenta un caso. Se analizan las teorías que hay acerca de su etiología, la clasificación y embriogénesis ${ }^{1,4}$.

\section{Summary}

Sirenomelia is a congenital malformation and is usually fatal because of associated malformations of unknown etiology without a hereditary pattern or proven genetic basis. It is produced by an early alteration in vascular development and the degree of defects depends on when that blood was hijacked during embryogenesis. Malformations may be associated with gastrointestinal, urinary tract and dextrocardia. In ancient siren-children were considered "monsters", and as such were killed or hidden by her family. Currently this type of malformation is easily detectable by laboratory tests and Cabinet who currently have. We present a case. It discusses the theories that are about the etiology, classification and embryogenesis.

Palabras clave: Sirenomelia, Malformación congénita. malformation.

Profesor Investigador y titular de la Materia Hematología UAA*

Estudiante del decimo semestre de la carrera de Medicina UAA ** 


\section{Introducción}

La Sirenomelia ha sido llamada también simpodia, simelia o feto sirenoide, Por la fusión de los miembros inferiores, similar a la cola de un pez, esta anomalía ha llamado la atención desde la antigüedad. Es posible que la leyenda de la sirenas, de la mitología griega, que eran mitad mujer, mitad pez, haya surgido de la observación de fetos sirenoides, los cuales fueron rodeados de toda clase de mitos, supersticiones y fantasías. Pinturas pertenecientes a los siglos $\mathrm{VI}$ y $V$, muestran ya figuras de sirenas (3). También se observan representaciones similares en leyendas hindúes y durante el imperio romano (Figura 1). La prevalencia de sirenomelia en la literatura, varía desde 1 en 50,000 hasta 1 en 100,000 nacimientos. Se presenta con mayor frecuencia en recién nacidos de sexo masculino, y una relación de 2.7 a 1 , con respecto al sexo femenino. De $8 \%$ a 15 $\%$ de los casos informados son gemelos monocigoticos (2).

La sirenomelia es una enfermedad extremadamente rara. Se produce por la fusión de los miembros inferiores secundaria a un trastorno severo en el desarrollo del blastema caudal axial posterior (en la cuarta semana de desarrollo embrionario - Figura 2), Los esbozos de los miembros inferiores aparecen a los 26 días, a nivel de las cuatro últimas somitas lumbares y de las tres primeras somitas sacras. La primera manifestación del desarrollo de las extremidades se encuentra a nivel del mesodermo lateral. La somatopleura de este mesodermo se engrosa y sus células pierden sus conexiones epiteliales y se transforman en una masa de mesénquima, sin que la somatopleura pierda su continuidad. Las acumulaciones de mesénquima son continuas y se disponen a lo largo de todo el cuerpo, ellas forman las llamadas crestas de Wolff. No obstante, solo en las regiones donde se desarrollan las extremidades superiores e inferiores continúan engrosadas, crecen y se transforman en el esbozo del miembro respectivo, Posiblemente debido a una alteración vascular de una rama de la arteria aorta abdominal. Se presenta de forma aislada o asociada a trastornos renales, cardiovasculares, gastrointestinales, respiratorios, neurológicos o genitales, formando parte del síndrome de regresión caudal, sin un patrón hereditario ni base genética demostrada (1-3) Esta insólita malformación se define por la fusión de las extremidades inferiores en mayor o menor grado, remedando así la figura de un pez. Valorada hasta ahora como la forma más severa de la regresión caudal, algunos autores tienden a considerarla independiente al no demostrarse una causa común entre ambas.

Figura 1, En esta otra imagen observamos a Odiseo pasando por la isla de las sirenas.

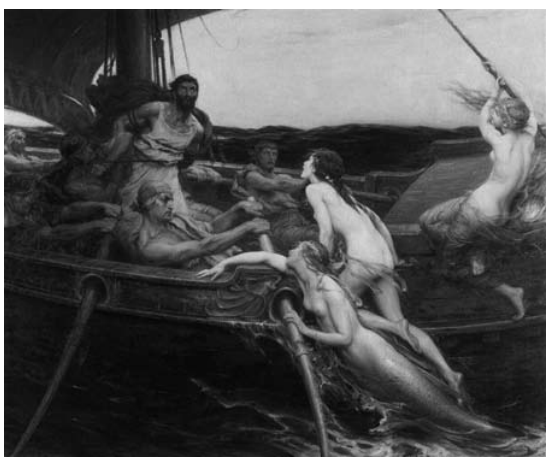




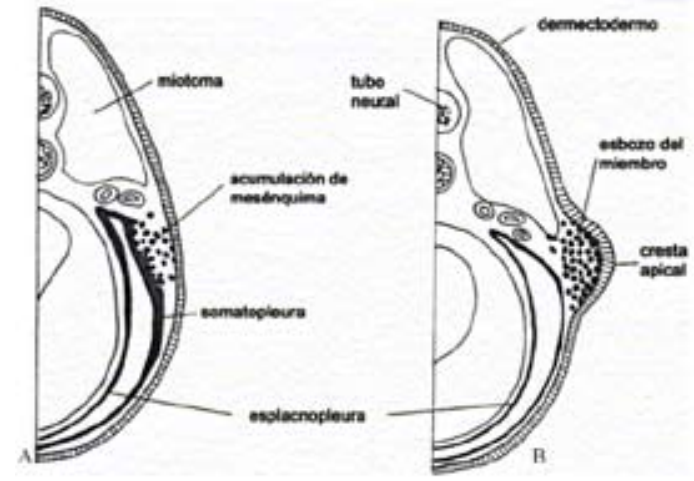

Figura 2, Esquema que representa el inicio del desarrollo del Miembro inferior. A: Engrosamiento de la Somatopleura y acumulación de mesénquima en el sitio de formación del Miembro Inferior. B: Desarrollo de la cresta Apical

\section{Clasificación}

Numerosos autores han tratado de agrupar los diferentes tipos de sirenomelia, pero aún no se ha logrado obtener una clasificación que sea internacionalmente aceptada.
Kampmeier refiere en su compendio, que en el siglo XIX algunos autores simplemente se refirieron a los vocablos: symelus, uromelus y sirenomelus; pero otros usaron la terminología de simpus dipus, simpus monopus y simpus apus, dependiendo si la extremidad inferior terminaba en dos pies, en un pie o en ninguno.

Aunque no se ha llegado aún a una clasificación del todo precisa algunos autores han tratado de generalizar esta malformación en sus clasificaciones, entre los más importantes podemos mencionar a: Duhamel realizó que una clasificación de acuerdo a la anatomía y patología hallada en los miembros inferiores. Según la gravedad de las anomalías, las agrupó en: A. formas más graves y $B$. formas menos graves (figura 3). Posteriormente, Stocker y Heifetz en $1987^{2,3}$, clasificaron la sirenomelia en siete variantes, los cuales se resumen en el Cuadro 1.

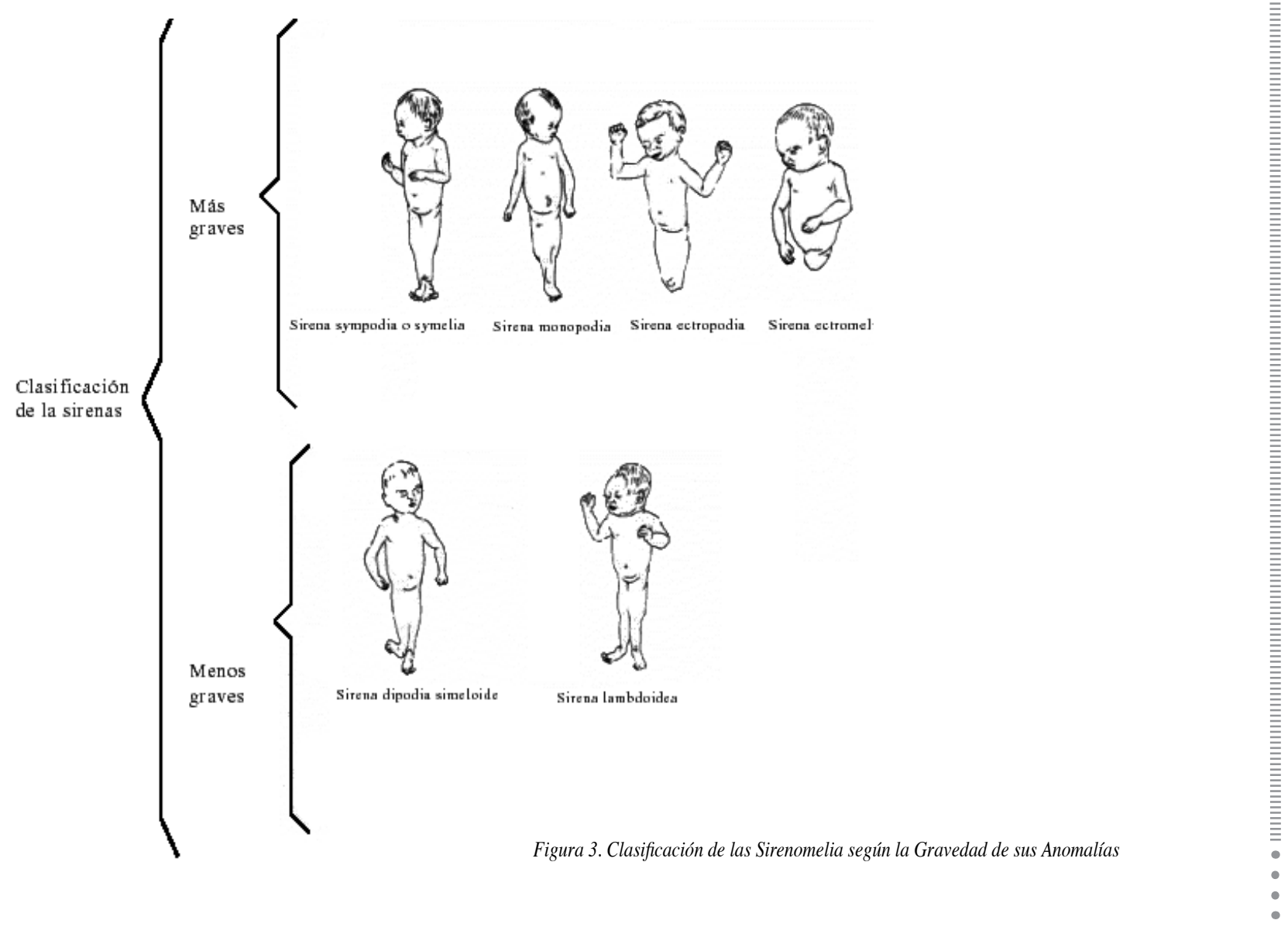




\section{Cuadro I}

\section{Clasificación de Stocker y Helfetz}

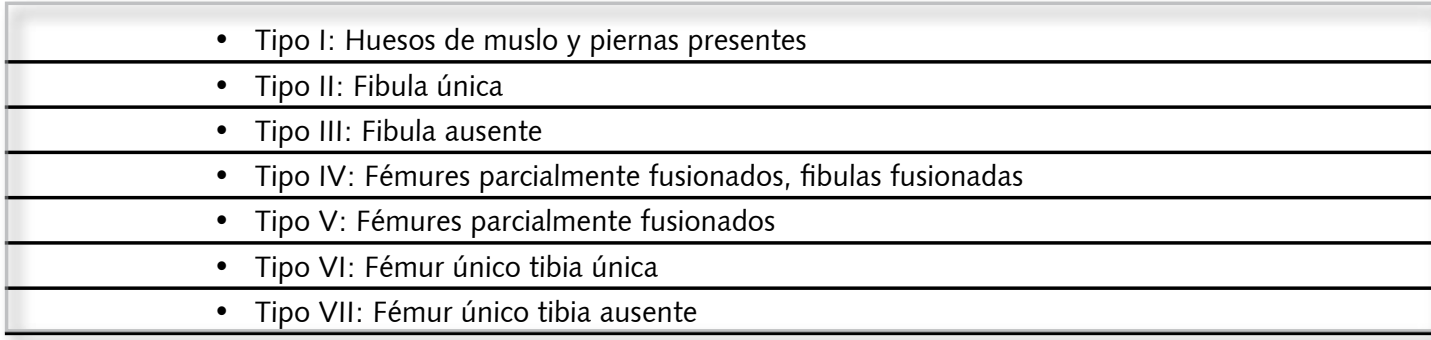

Fíbula = Peroné

\section{Presentación del caso}

Se trata de un producto vivo que nace a las 19:45 Hrs en medio hospitalario, hijo de madre de 20 años de edad, producto de gesta I, con amenaza de aborto en el 2 do trimestre, con control prenatal en 10 ocasiones. No se refieren otras patologías agregadas durante el embarazo, en us de control se encuentra oligohidramnios, por lo que se indica resolución de embarazo. Se obtiene producto único, vivo, intrauterino, por vía abdominal. Sexo indefinido (figura 4), pretérmino de 31 semanas de gestación según Capurro, pesó 1000 gramos. Talla $34.5 \mathrm{~cm}$, perímetro cefálico 27.5 $\mathrm{cm}$, perímetro torácico $21 \mathrm{~cm}$, perímetro abdominal $18.5 \mathrm{~cm}$, Apgar $3 / 5$. Silverman - Anderson 4 a los 5 minutos. Placenta y líquido amniótico, normales; cordón umbilical grueso con dos arterias y una vena.

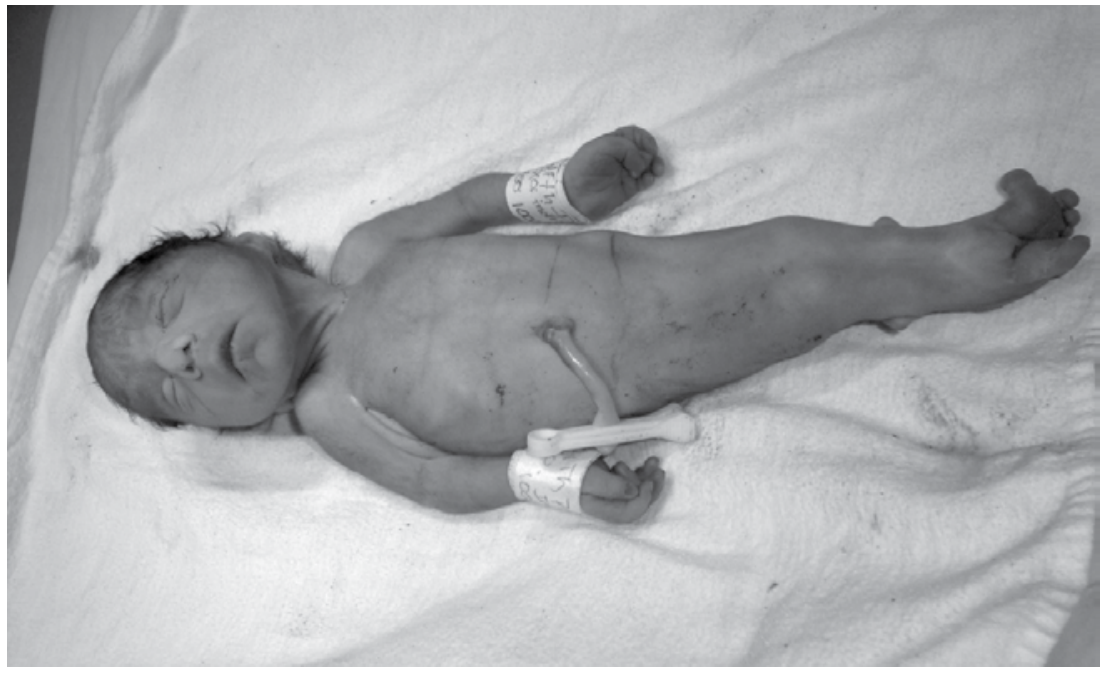

Figura 4. Producto con Sirenomelia. No se Observa genitales externos. 
Exploración física del producto: Se encuentra Implantación baja de pabellones auriculares con deformación de los mismos (figura 7), reflejo moro presente, coanas y esófago permeable, extremidades superiores anatómicamente íntegras, ausencia de glándula mamaria y genitales externos, cor- dón umbilical de aspecto normal, fusión de miembros inferiores con pies semicompletos y ausencia de perforación anal (Figura 5).

En la radiografía se observó ausencia de peroné y alteraciones en huesos de los pies (Figura 6) resto de exploración física aparentemente normal.

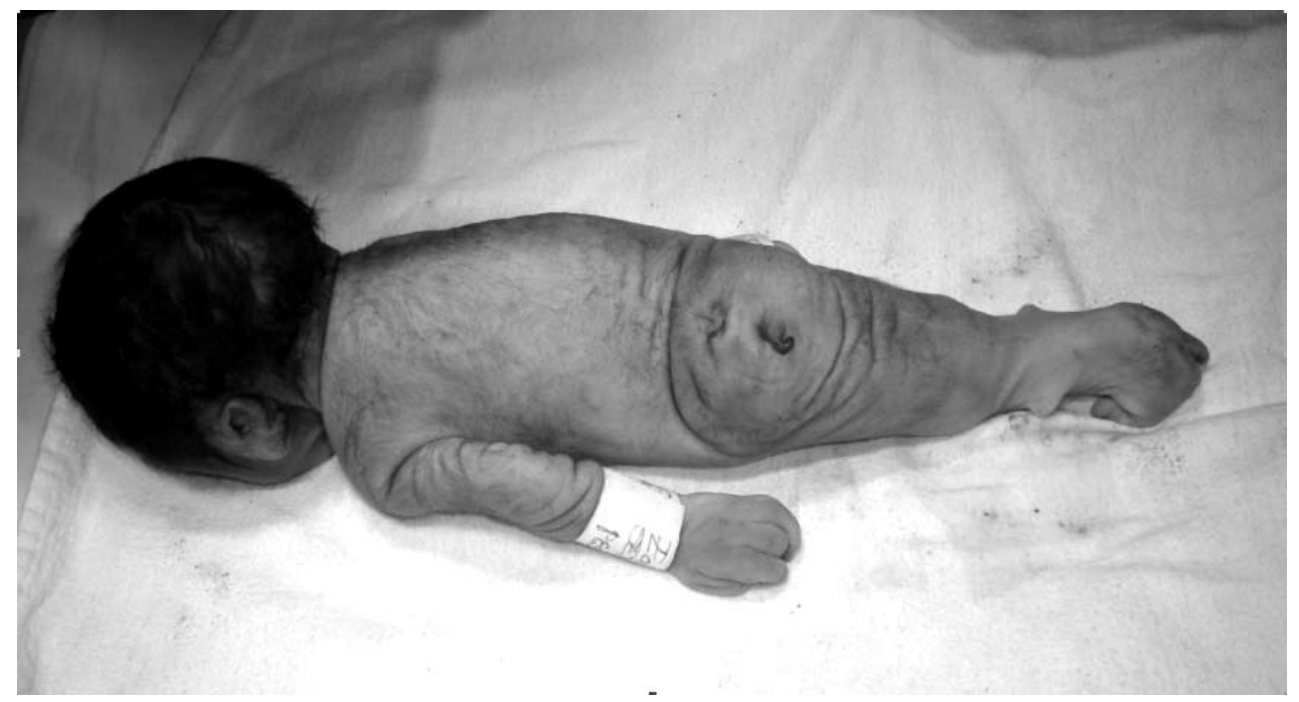

Figura 5. No se encuentra perforación Anal
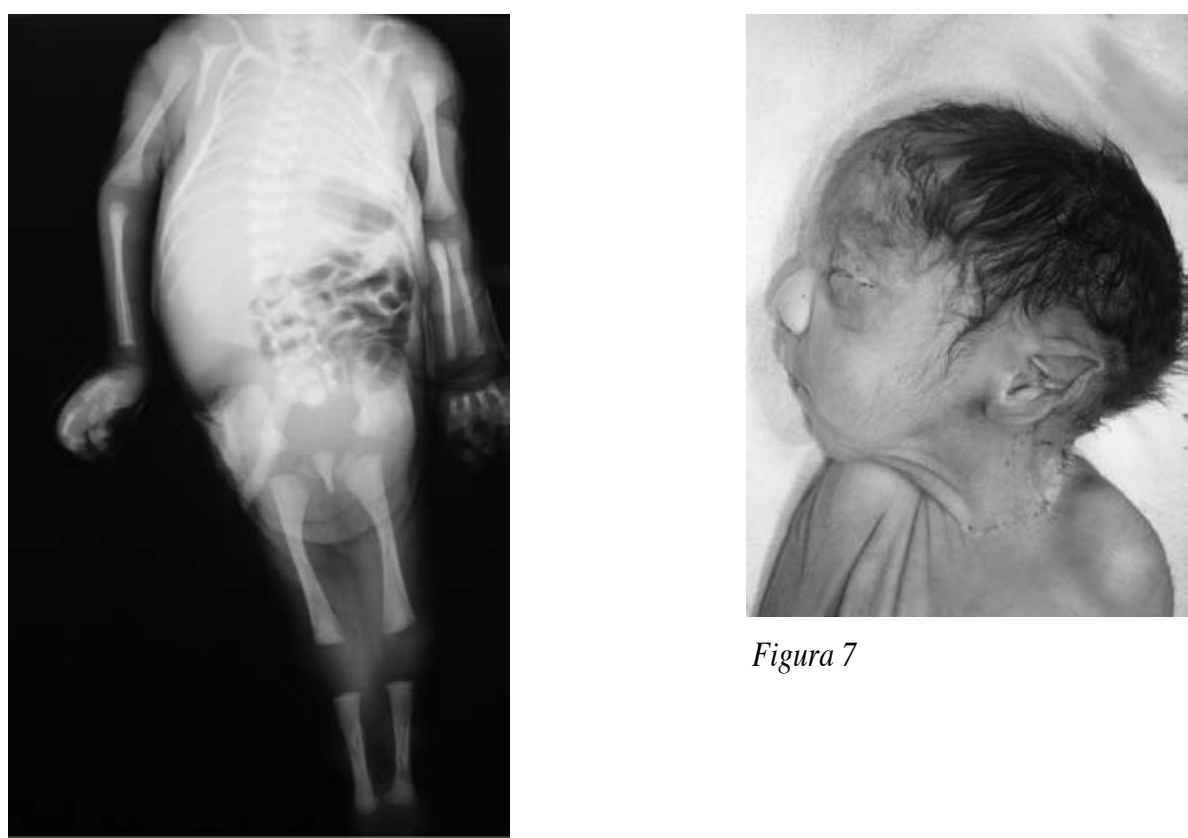

Figura 7

Figura 6 
También se encuentra hipoplasia pulmonar, agenesia renal, que sumados a los genitales ambiguos, atresia anal y sirenomelia se llega a la conclusión de que se trata de una secuencia de anomalías incompatibles con la vida, por lo que se le dan medidas paliativas, posteriormente el paciente presenta episodios de apnea y taquicardia/bradicardia y cae en paro cardiorespiratorio a las 22:35 Hrs, sufriendo defunción a esa misma hora.

\section{Diagnóstico}

El diagnóstico prenatal de la sirenomelia es muy importante, ya que por tratarse de un producto no viable, se podría plantear la interrupción oportuna del embarazo. El método utilizado para detectar este defecto en vida intrauterina es el ultrasonido. Durante el primer trimestre del embarazo, el feto está rodeado de suficiente cantidad de líquido amniótico que proviene casi en su totalidad de los amnioblastos de la membrana amniótica; por esta razón se hace más fácil la visualización de las alteraciones presentes. Durante el final del segundo trimestre del embarazo y comienzos del tercero, el oligohidramnios existente en la mayoría de los casos, puede entorpecer el diagnóstico de la sirenomelia, así como también la observación de las anomalías asociadas, como las del tubo neural, las cardíacas, las de la parte caudal de la columna vertebral y las del seno urogenital.

El diagnóstico de las características esquelética de los sirenomelos es más fácil realizarlo cuando existe un solo fémur y una sola tibia. Algunos autores refieren que utilizando la ultrasonografia transvaginal a la octava semana posmenstrual, pueden ser observados los miembros fetales. A la novena semana posmenstrual ya se pueden identificar el húmero y el fémur, mientras que el radio, el cúbito, la tibia y el peroné, sólo pueden ser vistos a la décima semana. Sin embargo, se consigue poca bibliografía en la que se indique el diagnóstico de la displasia esquelética, observada por ecosonografía en el primer trimestre del embarazo. Bronshtein y col, han referido el mayor número de anomalías esqueléticas estudiadas a finales del primero y comienzos del segundo trimestre del embarazo.

La utilización del Doppler a color ha permitido una mayor precisión en el diagnóstico de la sirenomelia y de las anomalías asociadas. Sepúlveda y col., utilizando este método identificaron en los casos de sirenomelia, una gran arteria que emerge de la aorta abdominal, la cual cursa centralmente hacia el cordón umbilical, ésta corresponde a la persistencia de una arteria vitelina. Este hallazgo puede ser considerado patognomónico para el diagnóstico prenatal de la sirenomelia. La ultrasonografía transvaginal en tercera dimensión, al igual que la ultrasonografía 3D en movimiento, también han sido utilizadas con éxito en el diagnóstico de la sirenomelia. Estas permiten corroborar las imágenes obtenidas con otros sonogramas y dan información adicional sobre los movimientos fetales y en general, sobre la condición y desarrollo del feto (3-4). 


\section{Discusión}

Formas más graves: incluyen aquellos casos en los cuales hay desde una fusión membranosa de los miembros, hasta una fusión total de los mismos, o reducción en forma de muñón y las severas malformaciones que se asocian a la sirenomelia, tanto internas como externas, hacen que generalmente ésta sea incompatible con la vida.

Si bien es una anomalía poco frecuente, cuyo mecanismo patogénico hasta el presente, no ha sido aclarado definitivamente es importante tomar en consideración las diversas hipótesis emitidas al respecto, ya que ellas pueden orientar sobre el origen de cada una de las anomalías presentes en los sirenomelos.

Es de suma importancia llegar a un diagnóstico prenatal conciso y preciso ya que de ello se toma el camino a seguir decidido por los padres en una situación determinada y así evitar complicaciones mayores. En la actualidad se tiene una amplia gama de exámenes por imagen como el doppler a color que nos pueden orientar con gran precisión a la observación de la anomalía en etapas tempranas del desarrollo y así tomar una mejor decisión. Cuando hay ausencia de genitales externos, hace indispensable que el sexo sea determinado por el examen histológico de las gónadas,

A pesar de que los informes de anomalías cromosómicas son ocasionales, siempre se debe realizar el cariotipo fetal, para descartarlas, y sobre todo se hace necesario realizar una historia clínica minuciosa y muy completa, con el fin de conocer la posible etiología de esta patología, y así evitar el riesgo que se repitan estas alteraciones en nuevos embarazos.

\section{Conclusiones}

Nuestro paciente de acuerdo con la clasificación de la gravedad de sus anomalías (Duhamel), lo clasificamos en: los más graves y a su vez subclasificado en sirena sympodia o symelia: caracterizado porque los miembros aparentemente completos, están incluidos dentro de una misma envoltura cutánea, los pies presentan las plantas hacia delante y están fusionados. Y según Stocker y Heifetz en Tipo III con Fíbula (Peroné) Ausente.

\section{Bibliografía}

1 Arturo Juárez Azpilcueta, Marco Antonio Durán PadiIla, Radames Rivas López, Luis Paulino. Islas Domínguez, Socorro Martínez Morales. Sirenomelia: Reporte de un caso de autopsia. Revista Mexicana de Pediatría. Vol. 72, Núm. 1 • Ene.-Feb. 2005. pp 21-23

2 Armicson Felipe Solano, m.d., Wilmar Saldarriaga, m.d., Carolina Isaza, m.d., m.sc., Pierpaolo Mastroiacovo, m.d., Eduardo e. C Astilla, m.d. Foco Epidémico de Sirenomelia en Cali, Colombia. Informe de 4 casos en el Hospital Universitario del Valle en 54 días. Colomb Med 2006; 37: 213-218 (Julio-Septiembre).

3 Drs. Agata Rodríguez M, María Luisa Carmona de Uzcátegui, Belkys Chacín P, Gerardo Tovitto, Adán Colina, Franklin Moreno. Revista de Obstetricia y Ginecología de Venezuela. Print ISSN 0048-7732. Rev Obstet Ginecol Venez vol.67 no.3 Caracas Sept. 2007
4 Miguel Lugones Botell, Luis Alberto Pichs García, Marieta Ramírez Bermúdez y Emilia Miyar Pieiga. Sirenomelia. Rev Cubana Med Gen Integr 2006;22(2)

5 Ponce de León TM. Sirenomelia. Bol Med. Hosp Infant Mex 2001;58: 862-65.

6 Patel S, Suchet I. The role of color and power Doppler ultrasound in the prenatal diagnosis of sirenomelia. Ultrasound Obstet Gynecol 2004; 24: 684-691.

7 Castilla EE, Orioli IM. ECLAMC: The Latin-American Collaborative Study of Congenital Malformations. Community Genet 2004; 7: 76-94.

8 Kjaer KW, Keeling JW, Opitz JM, Gilbert-Barness E, Hartling $U$, Hansen BF, et al. Sirenomelia sequence according to the distance between the first sacral vertebrae and the ilia. Am J Med Genet 2003; 120A: 503-508. 